\title{
Relationships between the superconducting gap, pseudogap and transition temperature in high- $\mathrm{T}_{\mathrm{c}}$ superconductors
}

\author{
M.Ya. Amusia ${ }^{a, b}$, V.R. Shaginyan ${ }^{a, c} *$ \\ ${ }^{a}$ The Racah Institute of Physics, the Hebrew University, Jerusalem 91904, Israel; \\ ${ }^{b}$ A.F. Ioffe Physical-Technical Institute, Russian Academy of Sciences, 194021 St. Petersburg, Russia; \\ c Petersburg Nuclear Physics Institute, Russian Academy of Sciences, Gatchina, 188350, Russia
}

\begin{abstract}
The crossover from superconducting gap to pseudogap is considered. We show that the superconductivity is destroyed at the temperature $T_{c}$, with the superconducting gap being smoothly transformed into the pseudogap. Relations, which are of general interest, between the maximum values of the gap $\Delta_{1}, T_{c}$ and $T^{*}$ are established. We presents arguments that $T^{*}$ as a function of the doping level $x$ is approximately a straight line that crosses the abscissa at some point $x_{F C}$. In the vicinity of this point $T^{*}$ coincides with $T_{c}$, and at $x=x_{F C}$ the fermion condensation quantum phase transition takes place.
\end{abstract}

PACS: 71.27.+a; 74.20.Fg; 74.25.Jb

Keywords: Fermion condensation; Superconductivity; Pseudogap

*E-mail: vrshag@thd.pnpi.spb.ru 
One of the most challenging problems of modern physics is the problem of systems with a big coupling constant. This problem is of crucial importance particularly in the quantum field theory, making even the quantum electrodynamics to be not a self-consistent theory [1]. The same problem persists in many-body physics. The Landau theory of normal Fermi liquids has offered a solution of this problem by introducing parameters which characterize the effective interaction into the theory [2]. Obviously, that the applicability of a theory that is described by big coupling constant is closely related to revealing its limitations. Usually, it is assumed that the breakdown of the Landau theory is defined by the Pomeranchuk stability conditions and occur when the Landau amplitudes being negative reach its critical value. Note that the new phase at which the stability conditions are restored can in principle be again described by the Landau theory. It was demonstrated rather recently [3] that the Pomeranchuk conditions are covering not all possible limitations: one is overlooked, being connected with the situation when, at temperature $T=0$, the effective mass can become infinitely big. As it was shown for model cases, it can happen leading to profound consequences. Indeed, it has been demonstrated that such a situation can take place provided the corresponding amplitude, or effective coupling constant, being positive reaches the critical value, producing a completely new class of strongly correlated Fermi liquids with the Fermion condensate (FC) [3, 4] which is separated from a normal Fermi liquid by the fermion condensation quantum phase transition (FCQPT) [5]. However, this state can appear only when we are dealing with the strong coupling limit where an absolutely reliable answer cannot be given, based on pure theoretical first principle ground. Therefore, the only way to check the mentioned above results is to consider experimental facts which can point to the existence of such a state. We assume that these facts can be find in the field of high- $T_{c}$ superconductors.

There is a number of experimental facts directly pointing to the existence of a pseudogap (PG) in the electronic excitation spectrum of high-temperature superconductors at temperatures above the critical temperature $T_{c}$ (see e.g. [6] for a recent review). This phenomenon may be attributed to the presence of Cooper pairs at temperatures $T \leq T^{*}$, while the long range phase coherence leading to the superconductivity manifests itself only at temperatures $T \leq T_{c}$ [7]. The other scenario suggests that the pseudogap has its origin in other then superconductivity phenomena [8]. Recent studies, based on the intrinsic tunneling spectroscopy, have revealed that the superconducting gap (SG) does vanish, while PG does not even change, at $T=T_{c}$ [9]. It is claimed that all this speaks in favor of different origin of two coexisting phenomena and against the precursor-superconductivity scenario of PG [9]. On the other hand, it was observed that the pseudogap phase is destroyed at any temperature by applying sufficiently strong magnetic fields [10], while a detailed examination of this experimental fact have shown that such a behavior is completely compatible with a superconducting origin of the pseudogap [11. Moreover, detailed examination of the tunneling spectra over wide doping range showed that PG at various doping levels of Bi-based high temperature superconductors is predominantly of superconducting origin and smoothly evolve from $\mathrm{SG}$ at $T \geq T_{c}$ 12 14. At present there is no consensus concerning the key question on whether PG has its origin in superconductivity. Cuprates are very complex materials and it is evident that PG may be caused by various reasons. Whatever the scenario of PG is taken, the accepted approach is to deal with a special case of PG and to explain the observed relations between the maximum value of the gap $\Delta_{1}$ at $T=0$ and temperatures $T_{c}$ and $T^{*}$. Observed experimentally [13, the very high ratios $2 \Delta_{1} / T_{c} \simeq 28$ and $T^{*} / T_{c} \simeq 7$, are hard to explain within the precursorsuperconductivity scenario, where, for instance, the ratio $T^{*} / T_{c}$ is not more then 1.2 [15]. By ruling out the possibility of the precursor-superconductivity scenario, one faces another problem: how to explain a simple relationship between $\Delta_{1}$ and $T^{*}$ that was found for various cuprates: $2 \Delta_{1} / T^{*} \simeq 4$ [13]. Combination of these striking experimental data present a challenging problem in the physics of high temperature superconductivity. 
In this Letter we propose a solution of this problem by using unorthodox model based on the combination of FCQPT [5] and the conventional theory of the superconductivity. We consider the crossover from the superconducting gap to the pseudogap, thus establishing relationships between the maximum value of the gap $\Delta_{1}$ and temperatures $T_{c}$ and $T^{*}$. We show that $T^{*}$ as a function of the doping level $x$ can be approximated by a straight line crossing the abscissa at some point $x_{F C}$. The function $T^{*}(x)$ and $T_{c}(x)$ coincides in the vicinity of the point $x=x_{F C}, x_{F C}$ being the point at which FCQPT takes place [5].

For the reader's convenience, we have to give here a summary of the most important points of the model [5,16] and start with a brief consideration of general properties of two-dimensional electron liquid in the superconducting state, when the system has undergone FCQPT. At $T=0$, the ground state energy $E_{g s}[\kappa(\mathbf{p}), n(\mathbf{p})]$ is a functional of the order parameter of the superconducting state $\kappa(\mathbf{p})$ and of the quasiparticle occupation numbers $n(\mathbf{p})$ and is determined by the known equation of the weak-coupling theory of superconductivity (see e.g. [17])

$$
E_{g s}=E[n(\mathbf{p})]+\int \lambda_{0} V\left(\mathbf{p}_{1}, \mathbf{p}_{2}\right) \kappa\left(\mathbf{p}_{1}\right) \kappa^{*}\left(\mathbf{p}_{2}\right) \frac{d \mathbf{p}_{1} d \mathbf{p}_{2}}{(2 \pi)^{4}} .
$$

Here $E[n(\mathbf{p})]$ is the ground-state energy of normal Fermi liquid, $n(\mathbf{p})=v^{2}(\mathbf{p})$ and $\kappa(\mathbf{p})=$ $v(\mathbf{p}) \sqrt{1-v^{2}(\mathbf{p})}$. It is assumed that the pairing interaction $\lambda_{0} V\left(\mathbf{p}_{1}, \mathbf{p}_{2}\right)$ is weak. Minimizing $E_{g s}$ with respect to $\kappa(\mathbf{p})$ we obtain the equation connecting the single-particle energy $\varepsilon(\mathbf{p})$ to $\Delta(\mathbf{p}, 0)$,

$$
\varepsilon(\mathbf{p})-\mu=\Delta(\mathbf{p}, 0) \frac{1-2 v^{2}(\mathbf{p})}{2 \kappa(\mathbf{p})} .
$$

The single-particle energy $\varepsilon(\mathbf{p})$ is determined by the Landau equation, $\varepsilon(\mathbf{p})=\delta E[n(\mathbf{p})] / \delta n(\mathbf{p})$ [2], and $\mu$ is the chemical potential. The equation for superconducting gap $\Delta(\mathbf{p}, T)$ takes form

$$
\Delta(\mathbf{p}, 0)=-\int \lambda_{0} V\left(\mathbf{p}, \mathbf{p}_{1}\right) \kappa\left(\mathbf{p}_{1}\right) \frac{d \mathbf{p}_{1}}{4 \pi^{2}}=-\frac{1}{2} \int \lambda_{0} V\left(\mathbf{p}, \mathbf{p}_{1}\right) \frac{\Delta\left(\mathbf{p}_{1}, 0\right)}{E\left(\mathbf{p}_{1}, 0\right)} \frac{d \mathbf{p}_{1}}{4 \pi^{2}} .
$$

Where $E(\mathbf{p}, T)=\sqrt{(\varepsilon(\mathbf{p})-\mu)^{2}+\Delta^{2}(\mathbf{p}, T)}$. If $\lambda_{0} \rightarrow 0$, then, one has $\Delta(\mathbf{p}, \mathbf{0}) \rightarrow 0$, and Eq. (2) reduces to that proposed in [3]

$$
\varepsilon(\mathbf{p})-\mu=0 \text {, if } \kappa(\mathbf{p}) \neq 0,(0<n(\mathbf{p})<1) ; \quad p_{i} \leq p \leq p_{f} \in L_{F C} .
$$

At $T=0$, Eq. (4) defines a new state of Fermi liquid with $\mathrm{FC}$ for which the modulus of the order parameter $|\kappa(\mathbf{p})|$ has finite values in $L_{F C}$ range of momenta $p_{i} \leq p \leq p_{f}$ occupied by FC, and $\Delta_{1} \rightarrow 0$ in $L_{F C}$ [3] 5]. Such a state can be considered as superconducting, with infinitely small value of $\Delta_{1}$ so that the entropy of this state is equal to zero. This state, created by the quantum phase transition, disappears at $T>0$ [5]. It follows from Eq. (4) that the system brakes into two quasiparticle subsystems: the first one in $L_{F C}$ range is occupied by the quasiparticles with the effective mass $M_{F C}^{*} \rightarrow \infty$, while the second by quasiparticles with finite mass $M_{L}^{*}$ and momenta $p<p_{i}$. If $\lambda_{0} \neq 0, \Delta_{1}$ becomes finite, leading to finite value of the effective mass $M_{F C}^{*}$ in $L_{F C}$, which can be obtained from Eq. (2) [5]

$$
M_{F C}^{*} \simeq p_{F} \frac{p_{f}-p_{i}}{2 \Delta_{1}}
$$

while the effective mass $M_{L}^{*}$ is disturbed weakly. Here $p_{F}$ is the Fermi momentum. It follows from Eq. (5) that the quasiparticle dispersion can be presented by two straight lines characterized by the effective 
masses $M_{F C}^{*}$ and $M_{L}^{*}$ respectively. These lines intersect near the binding energy $E_{0}$ of electrons which defines an intrinsic energy scale of the system:

$$
E_{0}=\varepsilon\left(\mathbf{p}_{f}\right)-\varepsilon\left(\mathbf{p}_{i}\right) \simeq \frac{\left(p_{f}-p_{i}\right) p_{F}}{M_{F C}^{*}} \simeq 2 \Delta_{1} .
$$

In fact, as it is seen from Eq. (5) and (6) even at $T=0, \mathrm{FC}$, being absorbed by the superconducting phase transition, never exhibits its feature which is the dispersionless plateau associated with $M_{F C} \rightarrow \infty$. As a result, FC forms a Fermi liquid characterized by the two finite effective masses and by the intrinsic energy scale $E_{0}$ [5].

FCQPT appears in a many-electron systems at relatively low density, when the effective interaction becomes sufficiently large [18]. Calculations based on simple models show that FC can exist as a stable state separated from the normal Fermi liquid by the phase transition [3]. In ordinary electron liquid this interaction is directly proportional to the dimensionless parameter $r_{s} \sim 1 / p_{F} a_{B}$, where $a_{B}$ is the Bohr radius. If $p_{i} \rightarrow p_{F} \rightarrow p_{f}$, Eq. (4) determines the point $r=r_{F C}$ at which FCQPT takes place, and $\left(p_{f}-p_{i}\right) / p_{F} \sim\left(r_{s}-r_{F C}\right) / r_{F C}$ [3]. FCQPT precedes the formation of charge-density waves or stripes, which take place at some value $r_{s}=r_{c d w}$ with $r_{F C}<r_{c d w}$, while the Wigner crystallization takes place even at larger values of $r_{s}$ and leads to insulator [18]. On the other hand, there are charge-density waves, or stripes, in underdoped copper oxides and finally at small doping levels one has insulators [19]. Then, in underdoped copper oxides, the line-shape of single-particle excitations strongly deviates from that of normal Fermi liquid, see, e.g. [6]. While, in the highly overdoped regime slight deviations from the normal Fermi liquid are observed [20]. Moreover, recent studies of photoemission spectra discovered an energy scale in the spectrum of low-energy electrons in cuprates, which manifests itself as a kink in the single-particle spectra [21,22]. The spectra in the energy range $(-200-0)$ meV can be described by two straight lines intersecting at the binding energy $E_{0} \sim(50-70) \mathrm{meV}$ [22]. All these peculiar properties are naturally explained within a model proposed in [5, 16. In our model, the doping level $x$ is regarded as the density of holes per unit cell. If $a$ is the separation between near neighbor $\mathrm{Cu}$ ions, then the area of unit cell is proportional to $a^{2}$, and $x / a^{2}$ is proportional to the hole density. We assume that $x_{F C}$ corresponds to the highly overdoped regime at which slight deviations from the normal Fermi liquid take place and introduce the effective coupling constant $g_{\text {eff }} \sim\left(x-x_{F C}\right) / x_{F C}$. According to the model, the doping level $x$ in cuprates is related to $r_{s}$ and to $\left(p_{f}-p_{i}\right)$ in the following way [5]:

$$
g_{e f f} \sim \frac{\left(x_{F C}-x\right)}{x_{F C}} \sim \frac{r_{s}-r_{F C}}{r_{F C}} \sim \frac{p_{f}-p_{i}}{p_{F}} .
$$

Here the value of $x=x_{F C}$ corresponding to $r_{F C}$ defines the point at which FCQPT takes place. We employ this model and Eq. (7) to consider relationships between the gap, pseudogap and $T_{c}$.

To solve Eq. (2) analytically, we take the Bardeen-Cooper-Schrieffer (BCS) approximation for the interaction [23]: $\lambda_{0} V\left(\mathbf{p}, \mathbf{p}_{1}\right)=-\lambda_{0}$ if $|\varepsilon(\mathbf{p})-\mu| \leq \omega_{D}$, and zero outside this domain, with $\omega_{D}$ being the characteristic phonon energy. Such an interaction gives good description of the maximum gap $\Delta_{1}$ in the case of the d-wave superconductivity, because the different regions with the maximum absolute value of $\Delta_{1}$ and the maximal density of states can be considered as disconnected [24]. Therefore, the gap in this region is formed by attractive phonon interactions which depend weakly on the momenta inside these regions. The gap becomes $p$-independent, $\Delta(\mathbf{p}, T)=\Delta_{1}(T), E(\mathbf{p}, T)=E(T)$ respectively, and Eq. (2) takes the form

$$
1=\lambda_{0} N_{F C} \int_{0}^{E_{0} / 2} \frac{d \xi}{E(0)}+\lambda_{0} N_{L} \int_{E_{0} / 2}^{\omega_{D}} \frac{d \xi}{E(0)}
$$


Here $\xi \equiv \varepsilon(\mathbf{p})-\mu$ and $N_{F C}$ is the density of states in $L_{F C}$, or $E_{0}$, range. As it follows from Eq. (5), $N_{F C}=\left(p_{f}-p_{F}\right) p_{F} / 2 \pi \Delta_{1}$. The density of states $N_{L}$ in the range $\left(\omega_{D}-E_{0} / 2\right)$ has the standard form $N_{L}=M_{L}^{*} / 2 \pi$. If $E_{0} \rightarrow 0$, Eq. (8) reduces to the BCS equation. Assuming that $E_{0} \leq 2 \omega_{D}$ and treating the second integral in the right hand side of Eq. (8) as a small perturbation of the solutions, we obtain 16

$$
\Delta_{1} \simeq 2 \beta \varepsilon_{F} \frac{p_{f}-p_{F}}{p_{F}} \ln (1+\sqrt{2})\left(1+\beta \ln \frac{2 \omega_{D}}{E_{0}}\right) \propto \varepsilon_{F}\left(x-x_{F C}\right)
$$

with the Fermi energy $\varepsilon_{F}=p_{F}^{2} / 2 M_{L}^{*}$, and dimensionless coupling constant $\beta=\lambda_{0} M_{L}^{*} / 2 \pi$. Here we use Eq. (7) to relate $\left(p_{f}-p_{F}\right) / p_{F}$ with the doping level $x$. Taking the usual values of the dimensionless coupling constant $\beta \simeq 0.3$, and $\left(p_{f}-p_{F}\right) / p_{F} \simeq\left(x_{F C}-x\right) \simeq 0.2$, we get from Eq. (9) very large value for $\Delta_{1} \sim 0.1 \varepsilon_{F}$, while in normal superconducting metals one has $\Delta_{1} \sim 10^{-3} \varepsilon_{F}$.

At finite temperatures, $T^{*} \leq T$, where $T^{*}$ is the minimum temperature at which $\Delta_{1}\left(T^{*}\right)=0$, Eqs. (5) and (6) are replaced by the following one [5]

$$
M_{F C}^{*} \simeq p_{F} \frac{p_{f}-p_{i}}{4 T} ; E_{0} \simeq 4 T
$$

To obtain the relationship between $T^{*}$ and $\Delta_{1}$, we use the generalization of Eq. (8) to finite temperatures. Then we put $T \rightarrow T^{*}$, that is $\Delta_{1}\left(T \rightarrow T^{*}\right) \rightarrow 0$, and have

$$
1=\lambda_{0} N_{F C} \int_{0}^{E_{0} / 2} \frac{d \xi}{\xi} \tanh \frac{\xi}{2 T}+\lambda_{0} N_{L} \int_{E_{0} / 2}^{\omega_{D}} \frac{d \xi}{\xi} \tanh \frac{\xi}{2 T} .
$$

Here, $M_{F C}^{*}$ and $E_{0}$ are given by Eq. (10). We take into account Eq. (9) and obtain from Eq. (11)

$$
2 \Delta_{1} / T^{*} \simeq 4
$$

Consider now the critical temperature $T_{c}$. One can define $T_{c}$ as the temperature when $\Delta_{1}\left(T_{c}\right) \equiv 0$, then, obviously $T_{c}=T^{*}$. Also, $T_{c}$ may be defined as a temperature at which the superconductivity vanishes. Thus, we have two different definitions, which in the case of the d-wave superconductivity can lead to different $T_{c}$ values because of the special behavior of the gap at the line of nodes [25]. Provided the pairing interaction $\lambda_{0} V\left(\mathbf{p}_{1}, \mathbf{p}_{2}\right)$ is the combination of attractive interaction and repulsive one, $V=V_{s r}+V_{l r}$, the d-wave superconductivity can take place, see e.g. [24]. We assume that the longrange component $V_{l r}$ of the pairing interaction is repulsive and has such radius $p_{l r}$ in the momentum space that $p_{F} / p_{l r} \leq 1$. The short-range component $V_{s r} \sim \lambda_{0}$ is relatively large and attractive, with its radius $p_{F} / p_{s r} \gg 1$. Now the gap depends on the angle $\theta$, which we reckon from the line of nodes of the gap, that is $\Delta(p, \theta=0, T)=0$. At finite temperatures, Eq. (3) takes form 17

$$
\Delta(\mathbf{p}, T)=-\frac{1}{2} \int \lambda_{0} V\left(\mathbf{p}, \mathbf{p}_{1}\right) \frac{\Delta\left(\mathbf{p}_{1}, T\right)}{E\left(\mathbf{p}_{1}, T\right)} \tanh \frac{E\left(\mathbf{p}_{1}, T\right)}{2 T} \frac{d \mathbf{p}_{1}}{4 \pi^{2}}
$$

Hereafter, we consider solutions of Eq. (13) in the vicinity of the line of nodes. We transform Eq. (13) by setting $p \approx p_{F}$ (because the gap $\Delta \neq 0$ only in the vicinity of the Fermi surface) and separating the contribution $I_{l r}$ coming from $V_{l r}$, from the contribution related to $V_{s r}$ and denoted as $I_{s r}$. At small angles $\theta, I_{l r}$ can be approximated by $I_{l r}=\theta A+\theta^{3} B$, with the parameters $A$ and $B$ being independent upon $T$, because they are defined by the integral over the regions occupied by FC. Thus, we have

$$
\Delta\left(p_{F}, \theta, T\right)=I_{s r}+I_{l r}=-\int_{0}^{2 \pi} \int V_{s r}\left(\theta, p_{1}, \phi_{1}\right) \kappa\left(p_{1}, \phi_{1}\right) \tanh \frac{E\left(p_{1}, \phi_{1}\right)}{2 T} \frac{p_{1} d p_{1} d \phi_{1}}{4 \pi^{2}}+\theta A+\theta^{3} B .
$$


Now we show that at temperatures above some temperature $T_{n}$, the solution of Eq. (14) has the second line of nodes at $\theta_{c}(T)$ in the vicinity of the line of nodes. To show this, we reduce Eq. (14) to an algebraic equation. We have $I_{s r} \sim\left(V_{s r} \Delta_{1} / T\right) \theta$ because $\tanh (E / 2 T) \approx E / 2 T$ for $E \ll T$ and $T \approx T_{n}$. Because of the small radius of $V_{s r}$ the integration in Eq. (14) runs over a small area located at the gap node. Dividing both parts of Eq. (12) by $\kappa(\theta)$, we obtain 25]

$$
E(\theta)=-\left(\frac{V_{0}}{T}-A_{1}-\theta^{2} B_{1}\right)|\theta|
$$

where $A_{1}=A / \kappa$ and $B_{1}=B / \kappa$ and $V_{0} \sim V_{s r}(0) \Delta_{1}$ is a constant. Imposing the condition that Eq. (13) has the only solution $\Delta \equiv 0$ when $V_{s r}=0$, we see that $A_{1}$ is negative. The factor in the brackets on the right-hand side of Eq. (15) changes its sign at some temperature $T_{n} \sim V_{0} / A_{1}$. Because the excitation energy must be $E(\theta)>0$, the sign of $\Delta$ must be reversed at the point $\theta=\theta_{c}$, with $\Delta\left(\theta_{c}\right)=\Delta(0)=0$. The gap $\Delta$ has an entire new line of nodes at $T \geq T_{n}$, with $\theta_{c} \rightarrow 0$ at $T \rightarrow T_{n}$, see Fig. 1 . The gap $\Delta(\theta)$ is very small in the region $\left[0<\theta<\theta_{c}\right]$ and can be destroyed only within this region because of the different reasons, for instance, by spin density waves or ferromagnetic fluctuations [25,26]. This extinction of the gap opens the channel for decay of the supercurrent. We can conclude that $T_{c} \sim T_{n}$ is the temperature at which the superconductivity vanishes, and a strong variation of the superconductivity characteristics may be observed when $T \rightarrow T_{n}$. At high levels of overdoping, that is $x \rightarrow x_{F C}$, FC plays unimportant role, the coefficient $A_{1}$ is small and $T_{n}$ has no sense because $T_{n}>T^{*}$. Therefore, $T_{c}=T^{*}$. As soon as the difference $\left(x-x_{F C}\right)$ increases, $T_{n}<T^{*}$, and we have $T_{c}<T^{*}$. Note, that even though $T_{c} \simeq T^{*}$, a pseudogap-like behavior can be observed because of the ratio $M_{L}^{*} / M_{F C}^{*} \ll 1$, which leads to a strong change in the density of states at $E_{0} \simeq 2 \Delta_{1}$, see Eq. (10). We conclude that at $T_{c} \leq T$ when pseudogap is small or absent, it is the scale $E_{0}$ that produces the pseudogap-like structure in the quasiparticle density of state at the energy $\simeq 2 \Delta_{1}$. In case of the presence of pseudogap, the strong change in the density of states is defined principally by the pseudogap, as it follows from the above consideration. At $T \leq T_{c}$, the superconducting peak emerges in the density of states. With decreasing temperature it moves to higher energies until it reaches its maximum value $2 \Delta_{1}$, merging with the kink in the density of states at $E_{0}$, as it was observed in the experiments [9].

At the underdoped levels, there are strong antiferromagnetic correlations, and the gap can be destroyed locally even at $T<T_{n}$, because the gap becomes small around its line of nodes at $\theta=0$. Therefore, in this domain of $x, T_{c}(x)<T_{n}(x)$ and the function $T_{c}(x)$ is no longer proportional to $\Delta_{1}$. As it follows from Eq. (9), one has $\Delta_{1} \sim\left(p_{f}-p_{i}\right) / p_{F} \sim\left(x_{F C}-x\right)$, and $\Delta_{1}$ continues to grow with the doping decreasing. A detailed analysis of this situation will be published elsewhere. At $T^{*}>T>T_{c}$, the gap occupies only a part of the Fermi surface, and SG smoothly transforms into the PG. As we have seen above, the ratio $2 \Delta_{1} / T_{c} \simeq 2 \Delta_{1} / T_{n}$ and can reach very high values. For instance, in the case of overdoped $\mathrm{Bi}_{2} \mathrm{Sr}_{2} \mathrm{CaCu}_{2} \mathrm{Q}_{6+\delta}$, where the superconductivity and the pseudogap are considered to be of the common origin, $2 \Delta_{1} / T_{c}$ is about 28 , while the ratio $2 \Delta_{1} / T^{*} \simeq 4$; this ratio is also equal to 4 in the case of various cuprates [13]. From Eq. (9) it follows that $\Delta_{1}$ is directly proportional to $\left(x_{F C}-x\right) \sim\left(p_{f}-p_{i}\right) / p_{F}$, and one finds from Eq. (12) that the function $T^{*}(x)$ presents approximately a straight line crossing the abscissa at the point $x \simeq x_{F C}$, while in the vicinity of this point $T^{*}(x)$ coincides with $T_{c}(x)$. Note, that at $\left(x-x_{F C}\right) \rightarrow 0$ the scale $E_{0} \rightarrow 0$ as well and the main contribution to the gap comes from the second term on the right-hand side of Eq. (8). This contribution, being obviously small, changes slightly the line around $x_{F C}$ point. Strong deviations from the straight line behavior can also take place in the case of strongly underdoped samples because of vanishing the superconductivity and approaching the insulator regime.

This work was supported in part by the Russian Foundation for Basic Research, No 01-02-17189. 


\section{REFERENCES}

[1] R.P. Feynman, QED The Strange Theory of Light and Matter, Princeton, New Jersey, Princeton University Press, 1985.

[2] L. D. Landau, Sov. Phys. JETP 3 (1956) 920.

[3] V.A. Khodel, V.R. Shaginyan, JETP Lett. 51 (1990) 553; V.A. Khodel, V.R. Shaginyan, V.V. Khodel, Phys. Rep. 249 (1994) 1.

[4] G. E. Volovik, JETP Lett. 53 (1991) 222.

[5] M.Ya. Amusia, V.R. Shaginyan, JETP Lett. 73 (2001) 232; M.Ya. Amusia, V.R. Shaginyan, Phys. Rev. B 63 (2001) 224507.

[6] T.Timusk, B. Statt, Rep. Prog. Phys. 62 (1999) 61.

[7] A.G. Loeser et al., Science 273 (1996) 325.

[8] S.-C. Zhang et al. Science 275 (1997) 1089.

[9] V.M. Krasnov, A. Yuregns, D. Winkler, Delsing, Claeson, Phys. Rev. Lett. 84 (2000) 5860; V.M. Krasnov, A.E. Kovalev, A. Yuregns, D. Winkler, Phys. Rev. Lett. 86 (2001) 2657.

[10] T. Shibauchi et. al., Phys. Rev. Lett. 86 (2001) 5763.

[11] P. Pieri, G.C. Strinati, D. Moroni, cond-mat/0203268.

[12] N. Miyakawa et al., Phys. Rev. Lett. 83 (1999) 1018.

[13] M. Kugler et al., Phys. Rev. Lett. 86 (2001) 4911.

[14] D.L. Feng et al., cond-mat/0108386; T. Sato et al., cond-mat/0108415.

[15] P.E. Lammert, D.S. Rokhsar, cond-mat/0108146.

[16] M.Ya. Amusia, S.A. Artamonov, V.R. Shaginyan, JETP Lett. 74 (2001) 435.

[17] D.R. Tilley, J. Tilley, Superfluidity and Superconductivity, Bristol, Hilger, 1975.

[18] V.A. Khodel, V.R. Shaginyan, M.V. Zverev, JETP Lett. 65 (1997) 253.

[19] G. Grüner, Density Waves in Solids, Addison-Wesley, Reading, MA, 1994.

[20] Z. Yusof et al., cond-mat/01044367.

[21] T. Valla et al., Science 285 (1999) 2110.

[22] P.V. Bogdanov et al., Phys. Rev. Lett. 85 (2000) 2581; A. Kaminski et al., Phys. Rev. Lett. 86 (2001) 1070.

[23] J. Bardeen, L.N. Cooper, J.R. Schrieffer, Phys. Rev. 108 (1957) 1175.

[24] A.A. Abrikosov, Phys. Rev. B 52 (1995) R15738; A.A. Abrikosov, cond-mat/9912394.

[25] V.R. Shaginyan, JETP Lett. 68 (1998) 527; M.Ya. Amusia, V.R. Shaginyan, Phys. Lett. A 259 (1999) 460.

[26] S.A. Artamonov, V.R. Shaginyan, JETP 92 (2001) 287. 


\section{FIGURES}

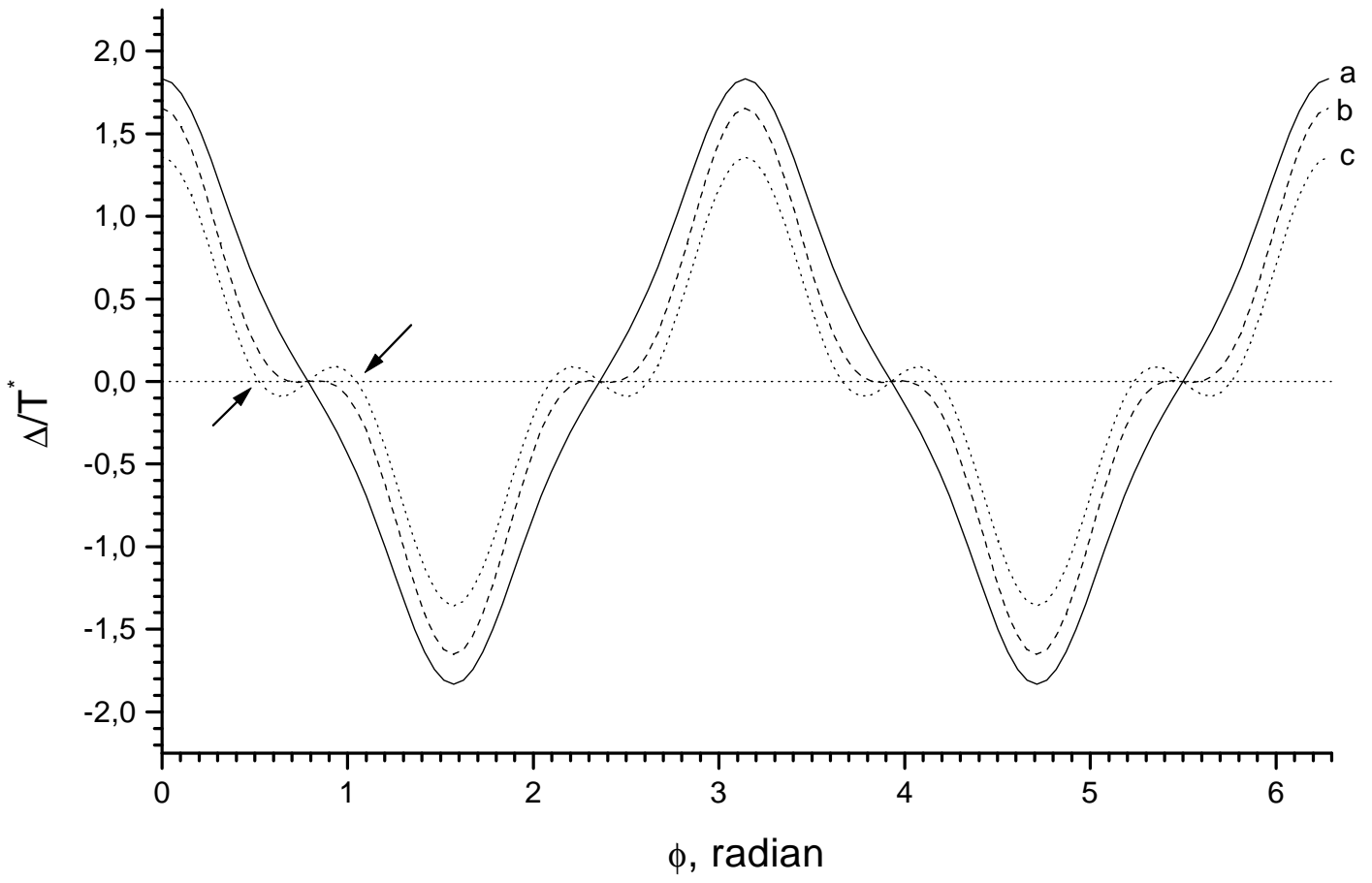

FIG. 1. The gap $\Delta$ as a function of $\phi=\theta+\pi / 4$ calculated at three different temperatures expressed in terms of $T_{n} \simeq T_{c}$, while $\Delta$ is presented in terms of $T^{*}$. Curve (a), solid line, shows the gap calculated at temperature $0.9 T_{n}$. In curve (b), dashed line, the gap is given at $T_{n} . \Delta(\phi)$ at $1.2 T_{n}$ is shown by curve (c), dotted line. The arrows indicate the two nodes at the Fermi surface emerged at $T_{n}$. 